\title{
Article \\ A Ubiquitous Clinic Recommendation System Using the Modified Mixed-Binary Nonlinear Programming-Feedforward Neural Network Approach
}

\author{
Yu-Cheng Lin $^{1}$ and Toly Chen ${ }^{2, *}$ \\ 1 Department of Computer-Aided Industrial Design, Overseas Chinese University, Taichung 40721, Taiwan; \\ yclin@ocu.edu.tw \\ 2 Department of Industrial Engineering and Management, National Yang Ming Chiao Tung University, \\ 1001 University Road, Hsinchu 300093, Taiwan \\ * Correspondence: tolychen@ms37.hinet.net
}

check for updates

Citation: Lin, Y.-C.; Chen, T. A Ubiquitous Clinic Recommendation System Using the Modified

Mixed-Binary Nonlinear

Programming-Feedforward Neural Network Approach. J. Theor. Appl.

Electron. Commer. Res. 2021, 16, 3282-3298. https://doi.org/ $10.3390 /$ jtaer16070178

Academic Editor: Doug Vogel

Received: 18 September 2021

Accepted: 22 November 2021

Published: 24 November 2021

Publisher's Note: MDPI stays neutral with regard to jurisdictional claims in published maps and institutional affiliations.

Copyright: (c) 2021 by the authors. Licensee MDPI, Basel, Switzerland. This article is an open access article distributed under the terms and conditions of the Creative Commons Attribution (CC BY) license (https:// creativecommons.org/licenses/by/ $4.0 /)$.

\begin{abstract}
Most of the existing ubiquitous clinic recommendation (UCR) systems adopt linear mechanisms to aggregate the attribute-level performances of a clinic to evaluate the overall performance. However, such linear mechanisms may not be able to explain the choices of all patients. To solve this problem, the modified mixed binary nonlinear programming (MMBNLP)-feedforward neural network (FNN) approach is proposed in this study. In the proposed methodology, first, the existing MBNLP model is modified to improve the successful recommendation rate using a linear recommendation mechanism. Subsequently, an FNN is constructed to fit the relationship between the attribute-level performances of a clinic and its overall performance, thereby providing possible ways to further enhance the recommendation performance. The results of a regional experiment showed that the MMBNLP-FNN approach improved the successful recommendation rate by $30 \%$.
\end{abstract}

Keywords: clinic; ubiquitous recommendation; mixed-binary nonlinear programming; feedforward neural network

\section{Introduction}

Rapid advancements in mobile communication and computer technologies have fostered numerous innovations in various fields such as recommender systems and medical services [1-4]. In medical services, a popular application is ubiquitous clinic recommendation (UCR), which is a smartphone application that recommends a nearby clinic to a patient for treatment by considering his/her condition and needs $[5,6]$. As in other recommendation systems, a patient may follow the recommendation result or may not [7]. Therefore, a UCR system must evolve itself to increase the percentage of patients following their recommendation results [8]. This issue has become more urgent during the COVID-19 pandemic because the capabilities of clinics in the diagnosis and treatment of COVID-19 are unequal [9], and there is a lack of relevant information [10].

Ubiquitous clinic recommendation is a challenging task because of the following reasons. First, unlike other types of recommendation systems, users of UCR systems (i.e., patients) may not be able to clearly express their needs [11,12]. In addition, due to the lack of information and background knowledge, it is difficult for users to communicate with each other, which makes the prevalent collaborative filtering (CF) technique useless [13]. Nevertheless, the quality of recommendation results is very important to users. To address this issue, a UCR system usually mines user preferences from historical data, thereby imitating their decision-making models to make recommendation results conform to user needs $[12,14,15]$.

Current challenges in UCR are discussed as follows. First, most existing methods apply linear rules to aggregate the attribute-level performances of a clinic to evaluate the overall performance. For example, Tung and Chang [16] applied a linear regression (LR) 
equation for this purpose. Chen [12] compared the advantages and disadvantages of three linear methods, including weighted average (WA), ordered weighted average (OWA), and fuzzy weighted average (FWA). The CF method adopted by Ekstrand et al. [13] measured the similarity between patients in a nonlinear way, but still aggregated the attribute-level performances linearly. In addition, theoretically, the relationship between the attributes of a clinic and its overall performance may be linear or nonlinear. Since a linear relationship is the special case of a nonlinear relationship, it is more inclusive to fit the relationship between the attributes of a clinic and its overall performance with a nonlinear function. However,

(1) There are various types of nonlinear relationships-high-order polynomials, $n$-th roots, exponents, logarithms, etc. However, there is little information about the suitability of each nonlinear function;

(2) The overall performance of a clinic has no actual value, making it difficult to apply supervised learning methods to learn from historical data.

To address these difficulties, this study sets the following objectives:

- Choosing a suitable nonlinear function to fit the relationship between the attributes of a clinic and its overall performance;

- Improving the successful recommendation rate using a UCR system.

To meet these objectives, the modified mixed-binary nonlinear programming (MMBNLP)feedforward neural network (FNN) approach is proposed in this study. The proposed methodology starts by modifying the existing mixed-binary nonlinear programming (MBNLP) model to improve the successful recommendation rate by applying a linear UCR rule. Subsequently, the actual value of the overall performance of a clinic is simulated. Then, an FNN is constructed to fit the relationship between the attribute-level performances of a clinic and its overall performance (i.e., the simulated actual value) to explain the choices of all patients.

The remaining sections of this paper are organized as follows. A survey of the literature is in Section 2. The proposed MMBNLP-FNN approach is introduced in Section 3 , including the procedure and a numerical example. To assess the performance using the MMBNLP-FNN approach, a region study was conducted in a small area of Taichung City, Taiwan, which is detailed in Section 4. The performance using the MMBNLP-FNN approach is also compared with those using several existing methods. Finally, some concluding remarks and directions for future investigation are given in the last section.

\section{Previous Work}

\subsection{Clinic Selection and Recommendation}

Some relevant references are reviewed below. In addition to references directly related to UCR, patients' considerations when choosing or evaluating clinics are also valuable information. When choosing a suitable clinic, patients usually consider factors such as reputation, atmosphere, staff (doctors and paramedical staff), location (hospital environment), and service costs $[13,14]$.

Simanski et al. [17] analyzed the considerations of patients who needed to relieve postoperative pain in choosing a suitable hospital. According to the results of a questionnaire analysis, the main factors affecting their choice included pain therapy, medical care by the physician, and nursing care.

Bae et al. [18] conducted a questionnaire survey to find out factors that dental patients considered when choosing a clinic. Cleanness and hygiene, equipment and facilities, career and word-of-mouth, staff kindness, ease of use, and clinic scale were determined to be the more influential factors. It was noteworthy that patients with different backgrounds (such as different ages, genders, etc.) emphasized different factors.

For a similar purpose, Lee [19] discovered that the basic elements of a dental clinic, traffic convenience, the physical environment of the dental clinic, and the explicit qualification of the dentist were the most critical factors to a patient's choice. However, for the 
purpose of UCR, a quantitative relationship between these critical factors and a patient's choice still needs to be established. Qualitative surveys are insufficient to address the needs of patients.

In the view of Zulqarnain et al. [20], a patient usually applies a multi-criteria decisionmaking method, such as the order preference by similarity ideal solution (TOPSIS) method [21], to select a suitable clinic. TOPSIS is more similar to a linear mechanism than a nonlinear mechanism. Factors considered by a patient include security, environment, qualified staff, and expenses. The implication of their study is that a clinic recommendation system can be established by aggregating these factors based on TOPSIS.

In the literature, there are a few applications of nonlinear methods to assess and compare clinics. For example, Lee et al. [22] constructed an FNN to classify patients according to their behavior in choosing hospitals. The disadvantage of this method was that a large amount of data needed to be collected to train the FNN. To this end, Lee et al. conducted a questionnaire survey. This method was time-consuming, uneconomical, and unsuitable for real-time or online applications.

The factors considered when choosing public hospitals and private hospitals are different [23]. In addition, patients may be concerned about different factors [15]. Dividing patients into groups is a feasible treatment for a UCR system. In addition, patients frequently share their experiences of medical consultation with each other. Therefore, $\mathrm{CF}$ techniques are also applicable.

Ghosh [24] conducted a questionnaire survey and concluded that the quality of treatment, referrals, transport convenience, cost, and safety and services were factors that can affect the selection of suitable hospitals in a large city in India.

Kutlu Gündoğdu et al. [25] proposed a fuzzy evaluation based on distance from average solution (fuzzy EDAS) method to solve a multi-criteria and multi-expert hospital selection problem for organ transplantation, in which all fuzzy variables and parameters were given in or approximated by hesitant fuzzy numbers.

According to the survey conducted by Alkadhi et al. [26], the intention of choosing a dentist will be affected by the information presented on social media, especially recommendations from friends and online reviews on high quality websites.

\subsection{Ubiquitous Clinic Recommendation}

UCR may be most valuable in regions where medical facilities are popular (for example, Taiwan). For regions with insufficient medical care, an important task is how to allocate limited medical resources among a large number of patients. UCR is also important for patients in unfamiliar areas.

Khoie et al. [27] conducted patient surveys, and then analyzed the survey results to divide patients into groups, evaluate the satisfaction level, and determine the factors that affected patient satisfaction. In this way, suitable hospitals could be recommended to certain groups of patients. However, a personalized recommendation was not possible.

From a similar perspective, Paranjay and Rajeshkumar [28] constructed various artificial neural networks (ANNs) to classify patients, thereby recommending suitable hospitals to similar patients.

Chao et al. [29] constructed a long short-term memory (LSTM) neural network to estimate the number of patients in each hospital at different times. In this way, patients could be recommended to different hospitals to avoid waiting. Burdens for doctors could also be averaged. This method is far from a personalized recommendation.

A UCR system should strive to imitate the decision-making mechanisms of patients when choosing clinics. In this regard, some past studies applied WA, FWA, or TOPSIS [6,20]. The weights of factors were derived from the preferences, recommendations, and choices of patients using methods such as INLP [30,31], NLP [11], response surface modeling (RSM) [31], etc. Recently, Chen and Chiu [12] divided patients into multiple groups according to their recorded preferences and choices. Then, for each patient group, a WA mechanism was established to make recommendations. For a new patient, the recommen- 
dation mechanisms of all groups were applied to make recommendations. However, it is not easy to describe the decision-making processes of patients using a simple linear model (i.e., WA). In order to overcome this difficulty, this research adopted a nonlinear model (i.e., FNN) instead.

During the COVID-19 pandemic, a number of ubiquitous medical recommendation systems emerged, such as apps for finding where to buy facial masks, reminding a user to wear a facial mask, or detecting whether a user is wearing a facial mask [32]. According to the statistics provided by Collado-Borrell et al. [33], most of the apps developed at this stage were used to provide information or news about COVID-19, record symptoms, and trace contacts.

Table 1 summarizes the differences between the MMBNLP-FNN approach and some existing methods. Compared with past studies, this research has the following novelties and contributions:

(1) This research uses a nonlinear function to fit the relationship between the attributes of a clinic and its overall performance, while past studies have usually used linear functions;

(2) This research establishes a mechanism to simulate the actual value of the overall performance of a clinic, so that the application of supervised learning methods such as FNN to UCR is possible.

Table 1. Differences between the MMBNLP-FNN approach and some existing methods.

\begin{tabular}{cccc}
\hline Method & $\begin{array}{c}\text { Type of } \\
\text { Mechanism }\end{array}$ & Model & $\begin{array}{c}\text { Explainability of } \\
\text { Patient Choices }\end{array}$ \\
\hline Tung and Chang [16] & Linear & LR & Partial \\
Ekstrand et al. [13] & Linear & CF & Partial \\
Chen [6] & Linear & FWA-INLP & Partial \\
Chen [11] & Linear & FWA-NLP & Partial \\
Chen and Chiu [31] & Linear & WA-NLP-RSM & Partial \\
Kutlu Gündoğdu et al. [25] & Linear & Fuzzy EDAS & Partial \\
Chen and Chiu [12] & Linear & WA-INLP (Group) & Partial \\
The MMBNLP-FNN approach & Nonlinear & MMBNLP-FNN & All \\
\hline
\end{tabular}

\section{The MMBNLP-FNN Approach}

\subsection{Procedure}

There are two stages of implementing the MMBNLP-FNN approach (see Figure 1). The first stage is model building that comprises the following steps:

Step 1. Perform an initial assessment of the overall performance of a clinic using the MMBNLP method.

Step 2. Adjust the assessment result to simulate the actual value.

Step 3. Construct an FNN to assess the overall performance of a clinic.

The second stage is the application that is composed of the following steps:

Step 4. (Patient) Access the UCR system using a dedicated app.

Step 5. (Central control unit) Record the location, required department, and preference of the patient into the system database.

Step 6. (Central control unit) Apply the trained FNN to make a recommendation that is recorded into the system database and transmitted to the patient.

Step 7. (Patient) Select a clinic and feedback his/her choice that is recorded into the system database. 


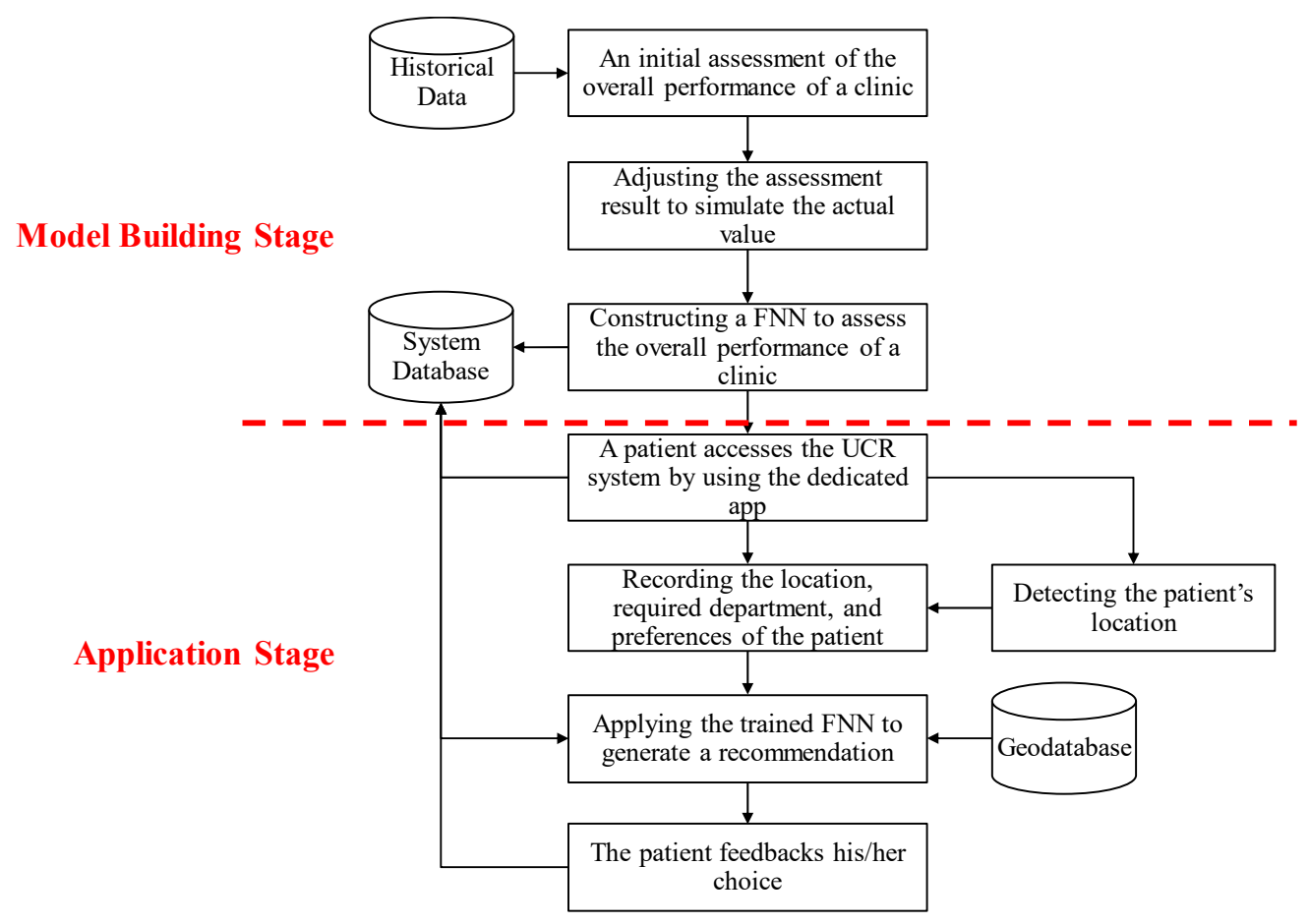

Figure 1. Implementation procedure of the MMBNLP-FNN approach.

\subsection{The MMBNLP Method}

The first step of the MMBNLP-FNN approach is to perform an initial assessment of the overall performance of a clinic using the following WA mechanism:

$$
\xi_{i j}=\frac{\sum_{q=1}^{Q} w_{q} \zeta_{i j q}}{\sum_{q=1}^{Q} w_{q}}
$$

where $\xi_{i j}$ is the overall performance of the $j$-th clinic considered by patient $i ; i=1 \sim n$ (the number of patients); $j=1 \sim m_{i}$ (the number of clinics). Therefore, the number of clinics considered by patients may vary. $\zeta_{i j q}$ is the $q$-th attribute-level performance of the clinic; $q=1 \sim Q$ (the number of attributes). $w_{q}$ is the weight assigned to attribute $q ; w_{q} \in[0,1]$. Under the assumption that $\sum_{q=1}^{Q} w_{q}=1$,

$$
\xi_{i j}=\sum_{q=1}^{Q} w_{q} \zeta_{i j q}
$$

The clinic that has the highest value of $\xi_{i j}$ will be recommended to patient $i$. However, the value of $w_{q}$ is unknown to the system administrator and must be estimated.

Let the clinic recommended to patient $i$ and the clinic chosen by him/her be denoted by $\alpha_{i}$ and $\beta_{i}$, respectively. $I_{i}$ is a state (binary) variable to represent whether the patient follows the recommendation:

$$
I_{i}=\left\{\begin{array}{cc}
1 & \text { if } \\
0 & \text { otherwise }
\end{array} \alpha_{i}=\beta_{i}\right.
$$


The successful recommendation rate $s$ can be evaluated as:

$$
s=\frac{1}{n} \sum_{i=1}^{n} I_{i}
$$

The clinic chosen by patient $i$ (i.e., clinic $\beta_{i}$ ) may not be superior to another clinic. A state (binary) variable $X_{i \beta_{i} j}$ is used to represent this. If $X_{i \beta_{i} j}=1$, clinic $\beta_{i}$ is better than clinic $j$; otherwise, the ordering of the two clinics is unknown, which can be represented as:

$$
\sum_{q=1}^{Q} w_{q} \zeta_{i \beta_{i} q} \geq X_{i \beta_{i} j} \sum_{q=1}^{Q} w_{q} \zeta_{i j q} ; j \neq \beta_{i}
$$

Patient $i$ considers $m_{i}$ clinics. Therefore, if clinic $\beta_{i}$ outperforms $m_{i}-1$ clinics, it is the top performing clinic that will be recommended to the patient. In other words, $\beta_{i}=\alpha_{i}$ if:

$$
\sum_{j \neq \beta_{i}} X_{i \beta_{i} j}=m_{i}-1
$$

or

$$
\frac{\sum_{j \neq \beta_{i}} X_{i \beta_{i} j}}{m_{i}-1}-1=0
$$

Equations (3) and (7) can be combined into:

$$
I_{i}\left(\frac{\sum_{j \neq \beta_{i}} X_{i \beta_{i} j}}{m_{i}-1}-1\right) \geq 0
$$

If $\sum_{j \neq \beta_{i}} X_{i \beta_{i} j}<m_{i}-1, I_{i}$ has to be 0 ; otherwise, $I_{i}$ will be 1 to maximize $s$.

To derive the optimal values of weights that maximize the successful recommendation rate, Chen and Chiu [31] solved the following MBNLP problem.

(MBNLP Problem)

$$
\operatorname{Max} s=\frac{1}{n} \sum_{i=1}^{n} I_{i}
$$

s.t.

$$
\begin{gathered}
\sum_{q=1}^{Q} w_{q} \zeta_{i \beta_{i} q} \geq X_{i \beta_{i} j} \sum_{q=1}^{Q} w_{q} \zeta_{i j q} ; j \neq \beta_{i} ; i=1 \sim n \\
\sum_{i}\left(\frac{\sum_{j \neq \beta_{i}} X_{i \beta_{i} j}}{m_{i}-1}-1\right) \geq 0 ; i=1 \sim n \\
\sum_{q=1}^{Q} w_{q}=1 \\
I_{i} \in\{0,1\} ; i=1 \sim n \\
X_{i \beta_{i} j} \in\{0,1\} ; j \neq \beta_{i} ; i=1 \sim n \\
w_{q} \in[0,1] ; q=1 \sim Q
\end{gathered}
$$

However, Chen and Chiu's method is unable to solve the following problems:

(1) In Constraint (10), the performance of clinic $\beta_{i}$ is likely to be exactly the same as that of a clinic it is superior to;

(2) There are many nonlinear constraints, which prolongs the solution time and reduces the possibility of reaching the global optimal solution. 
To solve these problems, the following treatments are taken in the proposed methodology:

(1) A threshold is added to Constraint (12) to break possible ties:

$$
\sum_{q=1}^{Q} w_{q} \zeta_{i \beta_{i} q} \geq X_{i \beta_{i} j}\left(\sum_{q=1}^{Q} w_{q} \zeta_{i j q}+\Delta\right) ; j \neq \beta_{i} ; i=1 \sim n
$$

(2) Constraint (5) is replaced by the following linear constraint:

$$
I_{i} \leq \frac{\sum_{j \neq \beta_{i}} X_{i \beta_{i} j}}{m_{i}-1} ; i=1 \sim n
$$

If clinic $\beta_{i}$ outperforms the other clinics, $\sum_{j \neq \beta_{i}} X_{i \beta_{i} j}=m_{i}-1$ and $I_{i}$ is 1 ; otherwise, $I_{i}$ has to be 0 .

As a result, the modified MBNLP (MMBNLP) model is proposed.

(MMBNLP Model)

$$
\operatorname{Max} s=\frac{1}{n} \sum_{i=1}^{n} I_{i}
$$

s.t.

$$
\begin{gathered}
I_{i} \leq \frac{\sum_{j \neq \beta_{i}} X_{i \beta_{i} j}}{m_{i}-1} ; i=1 \sim n \\
\sum_{q=1}^{Q} w_{q} \zeta_{i \beta_{i} q} \geq X_{i \beta_{i} j}\left(\sum_{q=1}^{Q} w_{q} \zeta_{i j q}+\Delta\right) ; j \neq \beta_{i} ; i=1 \sim n \\
\sum_{q=1}^{Q} w_{q}=1 \\
I_{i} \in\{0,1\} ; i=1 \sim n \\
X_{i \beta_{i} j} \in\{0,1\} ; j \neq \beta_{i} ; i=1 \sim n \\
w_{q} \in[0,1] ; q=1 \sim Q
\end{gathered}
$$

A numerical example is given in Table 2 to illustrate the application of the MMBNLP model.

Example 1. The example mentioned in Chen and Chiu [12] is modified to illustrate the proposed methodology. In this example, five patients, indicated with $A \sim E$, access the UCR system for recommending suitable clinics. For each patient, only the three clinics that are closest to the patient are considered. Therefore, $m_{i}=3 \forall i$. The attribute-level performances of the clinics are summarized in Table 2. The choices of the patients are shown in Table 3. To derive the values of weights in the WA mechanism that can explain patients' choices, the MMBNLP model is coded and optimized using Lingo. $\Delta=0.1$. The optimal solution is $\left\{w_{q}^{*}\right\}=\{0.1125,0.4500,0.4375\}$, giving $s^{*}=80 \%$. The recommendation results are summarized in Table 4.

Table 2. Illustrative example.

\begin{tabular}{cccc}
\hline $\boldsymbol{i}$ & $\left\{\zeta_{i 1 q}\right\}$ & $\left\{\zeta_{i 2 q}\right\}$ & $\left\{\zeta_{i 3 q}\right\}$ \\
\hline $\mathrm{A}$ & $(4,2,3)$ & $(2,3,3)$ & $(3,2,4)$ \\
$\mathrm{B}$ & $(5,3,4)$ & $(3,5,4)$ & $(4,4,3)$ \\
$\mathrm{C}$ & $(5,5,1)$ & $(5,4,2)$ & $(2,5,2)$ \\
$\mathrm{D}$ & $(4,3,3)$ & $(3,3,4)$ & $(1,5,1)$ \\
$\mathrm{E}$ & $(5,2,4)$ & $(4,3,4)$ & $(2,5,3)$ \\
\hline
\end{tabular}


Table 3. Choices of patients.

\begin{tabular}{lll}
\hline $\boldsymbol{i}$ & $\beta_{i}$ \\
\hline $\mathrm{A}$ & 3 \\
$\mathrm{~B}$ & 2 \\
$\mathrm{C}$ & 3 \\
$\mathrm{D}$ & 1 \\
$\mathrm{E}$ & 3 \\
\hline
\end{tabular}

Table 4. Recommendation results.

\begin{tabular}{lll}
\hline $\boldsymbol{i}$ & $\alpha_{\boldsymbol{i}}$ \\
\hline $\mathrm{A}$ & 3 \\
$\mathrm{~B}$ & 2 \\
$\mathrm{C}$ & 3 \\
$\mathrm{D}$ & 2 \\
$\mathrm{E}$ & 3 \\
\hline
\end{tabular}

\subsection{Simulating the Overall Performance of a Clinic}

From the results of the illustrative example, a simple, linear WA mechanism, even after optimization, is not able to explain the choices of all patients. To tackle this difficulty, a nonlinear decision-making (or recommendation) mechanism is required. To this end, an FNN is constructed in this study because a well-trained FNN can fit any complicated nonlinear relationship [34,35]. However, FNN is a supervised learning method that requires actual values, which is a challenging task since the overall performance of a clinic is a subjective concept and may differ from patient to patient. To address this issue, the proposed methodology adjusts the assessment result to simulate the actual overall performance of a clinic according to the following steps, so that the choices of all patients can be explained: Step 1 . Set $i=1$.

Step 2. If $\xi_{\beta_{i}} \leq \xi_{\alpha_{i}}$, adjust $\xi_{\beta_{i}}$ to $\xi_{\alpha_{i}}+\Theta$ and return to Step 1; otherwise, go to Step 3. $\Theta$ is the required amendment.

Step 3. Set $i=i+1$. If $i>n$, stop; otherwise, return to Step 2 .

The evaluation results in the previous example were adjusted to simulate the overall performances of clinics to explain the choices of all patients. The value of $\Theta$ was set to 0.2 . The simulation results are summarized in Table 5 . The choices of all patients can be explained since $\xi_{\beta_{i}} \geq \xi_{j}+\Delta \forall j \neq \beta_{i} . s=100 \%$.

Table 5. Simulated overall performances of clinics.

\begin{tabular}{llc}
\hline$i$ & $\xi_{\beta_{i}}$ \\
\hline A & 2.9875 \\
B & 4.3375 \\
C & 3.5857 \\
D & 3.6375 \\
E & 3.7875 \\
\hline
\end{tabular}

\subsection{FNN for Evaluating the Overall Performance of a Clinic}

The overall performance of a clinic is evaluated based on the attribute-level performances of the clinic. To this end, an FNN is constructed. The architecture of the FNN is illustrated in Figure 2. There are three layers in the FNN: the input layer, one hidden layer, and the output layer. There are $Q$ inputs to the FNN for each clinic. The number of nodes in the hidden layer is $R$. The FNN has one output that is the evaluated overall performance of clinic $j$. 


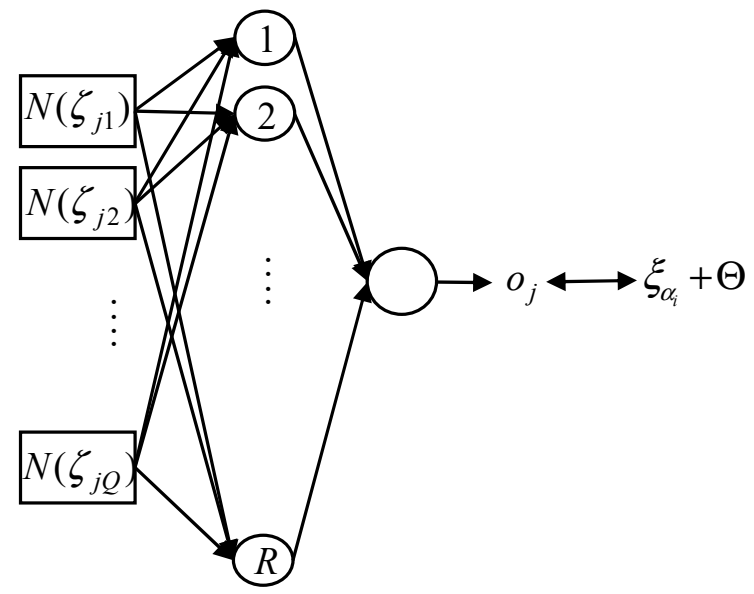

Figure 2. Architecture of the FNN.

Inputs to the FNN are the attribute-level performances of a clinic that are normalized using the partial normalization approach [36], so that the historical minimum and maximum, indicated with $\min _{\lambda} \zeta_{\lambda q}$ and $\max _{\lambda} \zeta_{\lambda q}$, respectively, can be exceeded in the future:

$$
\begin{gathered}
x_{j q}=N\left(\zeta_{j q}\right) ; q=1 \sim Q \\
N\left(\zeta_{j q}\right)=\frac{\zeta_{j q}-\min _{\lambda} \zeta_{\lambda q}}{\max _{\lambda} \zeta_{\lambda q}-\min _{\lambda} \zeta_{\lambda q}} \cdot 0.8+0.1
\end{gathered}
$$

$N()$ is the partial normalization function. $x_{j q}$ is the normalized value of $\zeta_{j q}$. Inputs are multiplied by weights, summated, and transferred to the hidden layer.

$$
I_{j r}^{h}=\sum_{q} w_{q r}^{h} x_{j q} ; r=1 \sim R
$$

where $w_{q r}^{h}$ is the connection weight between the input node $q$ and the hidden-layer node $r$. $I_{j r}^{h}$ is the input to the hidden-layer node $r$ for clinic $j$, which is compared with the threshold on the node $\left(\theta_{r}^{h}\right)$ :

$$
n_{j r}^{h}=I_{j r}^{h}-\theta_{r}^{h}
$$

and then is transformed and outputted as

$$
h_{j r}=\frac{1}{1+e^{-n_{j r}^{h}}}
$$

$h_{j r}$ is the output from hidden-layer node $r$ for clinic $j$, which is multiplied by the connection weight, summated, and transferred to the output layer:

$$
I_{j}^{o}=\sum_{r} w_{r}^{o} h_{j r}
$$

$I_{j}^{o}$ is the input to the output node, which is compared with the threshold on the output node $\left(\theta^{\circ}\right)$ :

$$
n_{j}^{o}=I_{j}^{o}-\theta^{o}
$$

Finally, the output from the FNN $o_{j}$ is generated as:

$$
o_{j}=\frac{1}{1+e^{-n_{j}^{o}}}
$$


which is un-normalized as

$$
U\left(o_{j}\right)=4 o_{i j}+1
$$

$U\left(o_{j}\right)$ will be compared with the simulated overall performance of the clinic. The FNN is trained as follows:

(1) Training algorithm: the Levenberg-Marquardt (LM) algorithm [37,38];

(2) Learning rate $(\eta): 0.1$;

(3) Number of epochs: 1000;

(4) Convergence criterion: mean squared error (MSE) $<10^{-4}$.

The FNN was applied to the previous example to evaluate the overall performance of a clinic. The evaluation results are shown in Figure 3. The FNN output was quite close to the simulated overall performance of the clinic. In this way, the overall performance of a clinic can be evaluated based on its attribute-level performances using the trained FNN.

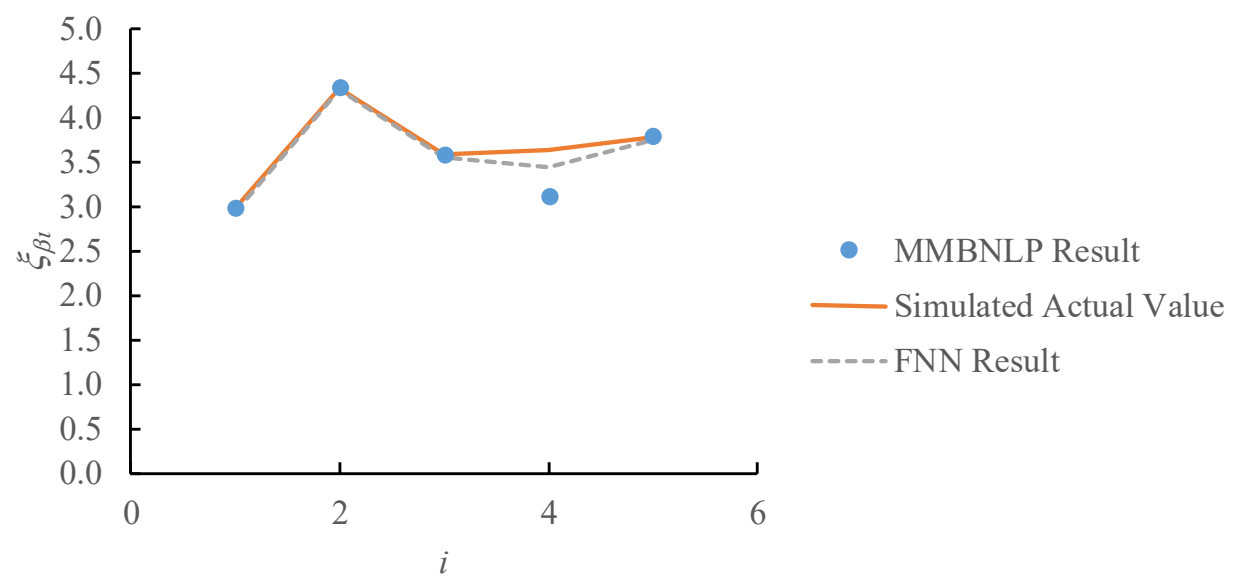

Figure 3. Fitting results using the FNN.

\section{Regional Experiment}

To evaluate the effectiveness of the proposed methodology, a regional study was conducted in a small area of Taichung City, Taiwan (see Figure 4). The experimental region was about $25 \mathrm{~km}^{2}$. There were more than 20 clinics in the experimental region, which are marked by red pushpins in the figure, providing medical services of various departments. Each clinic had four attributes: estimated waiting time, estimated travel time, preference for the clinic, and preference for the current doctor. To facilitate the subsequent aggregation, all attribute-level performances were normalized into $[1,5]$ according to the formulae in Table 6. The formulae used in this study were different from those used in Chen and Chiu [31]. Chen and Chiu [31] considered the minimum and maximum of data when evaluating clinic performances based on the estimated waiting time and travel time; however, they did not consider the minimum and maximum of another criterion, resulting in inconsistency and biased aggregation. 


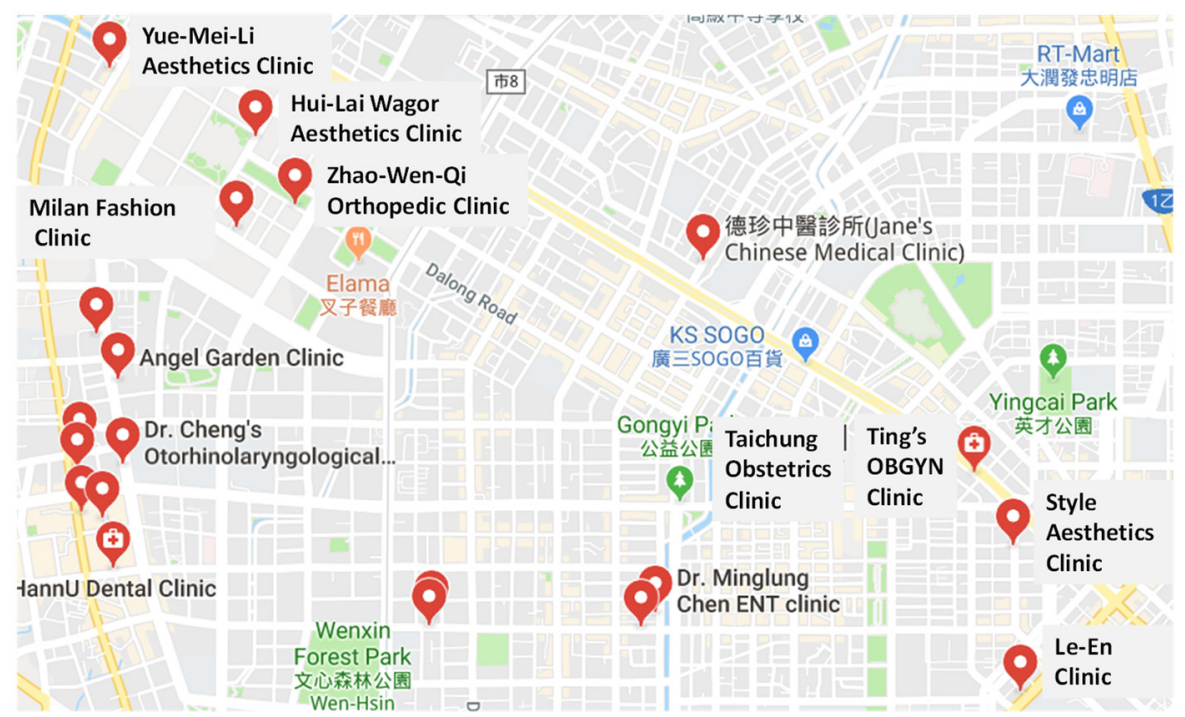

Figure 4. Experimental region.

Table 6. Criteria for assessing the attribute-level performances of a clinic.

\begin{tabular}{cl}
\hline \multicolumn{1}{c}{ Criteria } & Formulae for Assessing Attribute-Level Performances \\
\hline Estimated waiting time & $\zeta_{i j 1}=\left\lceil 4 \cdot \frac{x_{i j 1}-\min _{k, l} x_{k l 1}}{\max _{k, l} x_{k l 1}-\min _{k, l} x_{k l 1}}+1\right\rceil$ \\
& where $x_{i j 1}$ is the expected waiting time (in min) of patient $i$ \\
& after arriving at clinic $j$
\end{tabular}

According to the locations of patients, Google Maps was applied to search for nearby clinics. Table 7 summarizes the attribute-level performances of nearby clinics for the first 10 patients. Table 8 lists the clinics selected by the patients. 
Table 7. Performances of nearby clinics for the first 10 patients.

\begin{tabular}{cccc}
\hline$i$ & $\zeta_{i 1}$ & $\zeta_{i 2}$ & $\zeta_{i 3}$ \\
\hline 1 & $(2,3,2,3)$ & $(4,4,2,5)$ & $(1,4,3,1)$ \\
2 & $(2,2,3,4)$ & $(4,5,1,2)$ & $(2,4,2,1)$ \\
3 & $(5,2,2,4)$ & $(4,4,2,4)$ & - \\
4 & $(2,4,4,4)$ & $(3,1,3,5)$ & - \\
5 & $(3,5,3,2)$ & $(3,2,1,4)$ & $(2,2,2,2)$ \\
6 & $(3,3,4,4)$ & $(2,2,2,3)$ & - \\
7 & $(2,5,5,2)$ & $(5,4,4,3)$ & - \\
8 & $(3,3,4,4)$ & $(4,5,4,1)$ & $(3,2,4,4)$ \\
9 & $(4,5,2,2)$ & $(2,4,4,3)$ & $(4,4,2,1)$ \\
\hline
\end{tabular}

Table 8. Clinics selected by patients.

\begin{tabular}{ll}
\hline$i$ & $\beta_{i}$ \\
\hline 1 & 2 \\
2 & 2 \\
3 & 1 \\
4 & 1 \\
5 & 1 \\
6 & 1 \\
7 & 2 \\
8 & 1 \\
9 & 1 \\
10 & 2 \\
\hline
\end{tabular}

Based on their choices, the MMBNLP problem was formulated and solved, which resulted in the following WA mechanism:

$$
\xi_{i j}=0.195 \zeta_{i j 1}+0.102 \zeta_{i j 2}+0.220 \zeta_{i j 3}+0.483 \zeta_{i j 4}
$$

If this WA mechanism was applied to recommend suitable clinics to these patients, the results are shown in Table 9. The successful recommendation rate (s) was only $50 \%$. Obviously, a linear decision-making/recommendation mechanism such as WA was not sufficient to explain the decisions of all patients. To solve this problem, an FNN was constructed as a nonlinear decision-making/recommendation mechanism. First, the overall performances of clinics evaluated using WA were adjusted to simulate the actual values that could explain the choices of all patients. The results are summarized in Table 10.

Table 9. Clinics recommended to patients.

\begin{tabular}{ll}
\hline $\boldsymbol{i}$ & $\alpha_{i}$ \\
\hline 1 & 2 \\
2 & 1 \\
3 & 2 \\
4 & 2 \\
5 & 2 \\
6 & 1 \\
7 & 2 \\
8 & 1 \\
9 & 3 \\
10 & 2 \\
\hline
\end{tabular}


Table 10. Simulated overall performances.

\begin{tabular}{cccc}
\hline $\boldsymbol{i}$ & $\xi_{i 1}$ & $\xi_{i 2}$ & $\xi_{i 3}$ \\
\hline 1 & 2.585 & 4.043 & 1.746 \\
2 & 3.186 & 3.386 & 1.721 \\
3 & 3.760 & 3.560 & - \\
4 & 3.960 & 3.762 & - \\
5 & 3.141 & 2.941 & 2.000 \\
6 & 3.703 & 2.483 & 3.458 \\
7 & 2.966 & 3.712 & - \\
8 & 3.703 & 2.653 & - \\
9 & 3.801 & 3.127 & 3.601 \\
10 & 2.111 & 2.365 & 2.111 \\
\hline
\end{tabular}

Subsequently, an FNN was constructed to fit the relationship between the attributelevel performances of a clinic and its simulated overall performance. The fitting results are shown in Figure 5. Obviously, the FNN provided a very good fit: there were only four clinics with small deviations between the outputs and the simulated overall performances.

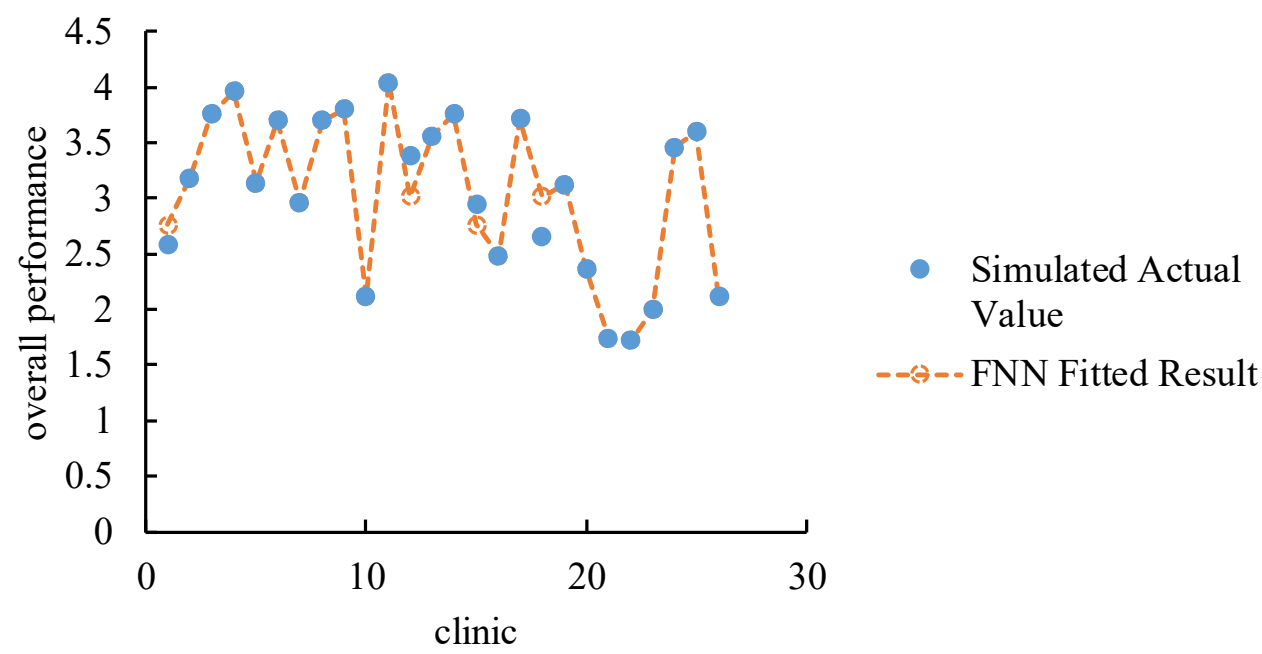

Figure 5. Fitting results.

If the FNN was applied to recommend suitable clinics to patients, the successful recommendation rate (s) would be $90 \%$.

Subsequently, both the fitted WM mechanism and the trained FNN were applied to patients 11 20 whose data were not learned. Table 11 summarizes the attribute-level performances of the clinics considered by these patients. Table 12 lists the clinics recommended to these patients using the WM mechanism and their subsequent choices. The successful recommendation rate was $70 \%$. To apply the trained FNN, the attribute-level performances of each clinic were fed into the FNN to derive its overall performance. After comparing the overall performances of all clinics, the top performing clinic was recommended to the patient. The recommendation results are summarized in Table 13. The successful recommendation rate was elevated to $90 \%$. 
Table 11. Attribute-level performances of clinics considered by patients 11-20.

\begin{tabular}{cccc}
\hline$i$ & $\zeta_{i 1}$ & $\zeta_{i 2}$ & $\zeta_{i 3}$ \\
\hline 11 & $(1,4,4,2)$ & $(3,2,4,4)$ & $(1,3,3,5)$ \\
12 & $(1,2,4,4)$ & $(3,3,2,4)$ & $(3,5,5,2)$ \\
13 & $(2,4,2,2)$ & $(3,2,2,4)$ & $(1,5,4,4)$ \\
14 & $(5,3,3,5)$ & $(2,5,3,5)$ & $(4,1,3,1)$ \\
15 & $(3,4,4,1)$ & $(3,3,1,3)$ & $(3,2,2,5)$ \\
16 & $(3,4,3,4)$ & $(5,2,4,3)$ & $(3,3,1,2)$ \\
17 & $(2,2,2,3)$ & $(3,3,1,4)$ & $(1,1,3,1)$ \\
18 & $(3,4,3,4)$ & $(4,4,1,2)$ & - \\
19 & $(1,1,4,4)$ & $(2,2,5,2)$ & - \\
20 & $(5,3,2,5)$ & $(3,3,1,1)$ & \\
\hline
\end{tabular}

Table 12. Recommended clinics to patients 11-20 using the WM mechanism.

\begin{tabular}{ccc}
\hline $\boldsymbol{i}$ & $\boldsymbol{\alpha}_{\boldsymbol{i}}$ & $\boldsymbol{\beta}_{\boldsymbol{i}}$ \\
\hline 11 & 2 & 2 \\
12 & 2 & 3 \\
13 & 3 & 3 \\
14 & 1 & 1 \\
15 & 3 & 3 \\
16 & 1 & 2 \\
17 & 3 & 3 \\
18 & 1 & 1 \\
19 & 1 & 2 \\
20 & 1 & 1 \\
\hline
\end{tabular}

Table 13. Recommendation results using the trained FNN.

\begin{tabular}{ll}
\hline $\boldsymbol{i}$ & $\boldsymbol{\alpha}_{\boldsymbol{i}}$ \\
\hline 11 & 2 \\
12 & 3 \\
13 & 3 \\
14 & 1 \\
15 & 3 \\
16 & 2 \\
17 & 3 \\
18 & 1 \\
19 & 1 \\
20 & 1 \\
\hline
\end{tabular}

According to the experimental result, the following discussion was developed:

(1) After optimization, the successful recommendation rate using the WM mechanism was only fair- $70 \%$, which showed that the decision-making mechanisms of patients might not be fully described by a simple linear mechanism such as WM.

(2) Compared with the WM mechanism, the trained FNN achieved a better performance in elevating the successful recommendation rate,

(3) To further elaborate on the effectiveness of the MMBNLP-FNN approach, three existing methods, including WA, OWA, and Chen and Chiu's method, were also applied to the collected data for comparison. In WA, the values of weights were the same as those derived using Equation (34) for fair comparison. In OWA, the moderately optimistic decision strategy was adopted, in which weights of $0.6598,0.1525,0.1051$, and 0.0827 were assigned to the first, second, third, and fourth performing attributes, respectively. In Chen and Chiu's method, the MBNLP problem was solved to determine the values of weights. Figure 6 shows the comparison results of the performances using various methods. Obviously, the MMBNLP-FNN approach outperformed the existing 
methods by achieving the highest successful recommendation rate. In addition, the proposed methodology elevated the successful recommendation rates for both the training and test data. The advantage of the proposed methodology over WA was the most significant (i.e., up to $30 \%$ ).

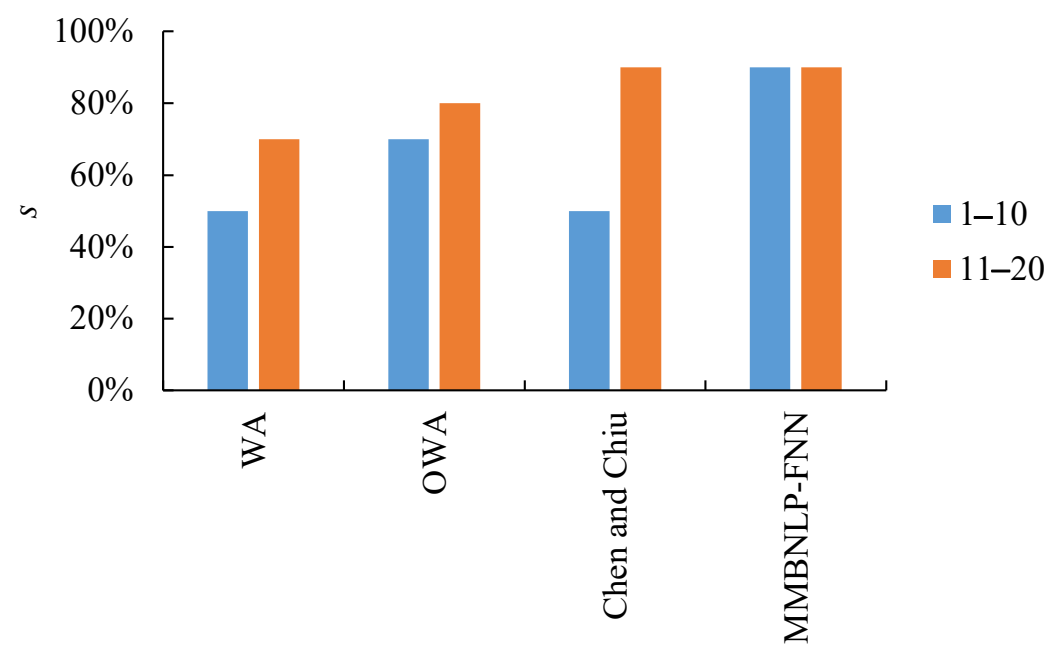

Figure 6. Performances using various methods.

\section{Conclusions}

UCR is a unique location-based service that has benefitted from advances in mobile communication and computer technologies. The major problem facing existing UCR systems is the ineffectiveness of linear mechanisms, such as WA, OWA, FWA, LR, or CF, in aggregating the attribute-level performances of a clinic to evaluate the overall performance. However, such a linear mechanism is too simple to explain the choices of all patients. To address this issue, the MMBNLP-FNN approach was proposed in this study. The MMBNLP-FNN approach first modifies the existing MBNLP model to further optimize the values of weights in the WA rule. Then, the overall performances of clinics are simulated to explain the choices of all patients. Finally, an FNN is constructed to fit the relationship between the attribute-level performances of a clinic and its overall performance.

A numerical example was given herein to illustrate the applicability of the MMBNLPFNN approach. Moreover, a regional study was conducted to elaborate the effectiveness of the proposed methodology. The results of the regional study indicate the following:

(1) The existing linear mechanism could be improved by optimizing the values of weights. To this end, the proposed MMBNLP model was effective;

(2) The trained FNN further elevated the successful recommendation rate using the UCR system, which showed that nonlinear rules could better describe the decision-making mechanisms of patients;

(3) The advantage of the proposed methodology over WA was the most obvious, in which the successful recommendation rate was elevated by $30 \%$. The superiority over Chen and Chiu's MBNLP model was also significant.

The FNN architecture created in this study is simple. In the future, more sophisticated architectures of FNN could be designed, such as an FNN ensemble [39] or a deep FNN $[40,41]$, to further enhance the interpretability of the UCR system. In addition, the uncertainty inherent in the decision-making process of a patient could be considered by replacing the FNN by a fuzzy ANN [42-45], which provides another direction for future investigation. 
Author Contributions: Both authors contributed equally to the writing of this paper. Both authors read and approved the final manuscript. Data curation, methodology and writing original draft: T.C. and Y.-C.L.; writing-review and editing: T.C. and Y.-C.L. All authors have read and agreed to the published version of the manuscript.

Funding: This research received no external funding.

Institutional Review Board Statement: Not applicable.

Informed Consent Statement: Informed consent was obtained from all subjects involved in the study.

Data Availability Statement: The study did not report any data.

Acknowledgments: This work was supported by Ministry of Science of Technology of Taiwan.

Conflicts of Interest: The authors declare no conflict of interest.

\section{References}

1. Kupperman, E.; Vigil, D.; Yazdani, A.; Baldwin, K. Model of Current Practice Regarding Prescriptions of Controlled Substances and the Perceived Benefits of E-Prescribing in an Academic Medical Center. J. Med. Syst. 2016, 40, 278. [CrossRef] [PubMed]

2. Baumel, A.; Birnbaum, M.L.; Sucala, M. A Systematic Review and Taxonomy of Published Quality Criteria Related to the Evaluation of User-Facing eHealth Programs. J. Med. Syst. 2017, 41, 128. [CrossRef] [PubMed]

3. Shah, Y.; Dunn, J.; Huebner, E.; Landry, S. Wearables data integration: Data-driven modeling to adjust for differences in Jawbone and Fitbit estimations of steps, calories, and resting heart-rate. Comput. Ind. 2017, 86, 72-81. [CrossRef]

4. Albahri, O.S.; Mohammed, K.I.; Zaidan, A.A.; Zaidan, B.B.; Hashim, M.; Salman, O.H. Systematic Review of Real-time Remote Health Monitoring System in Triage and Priority-Based Sensor Technology: Taxonomy, Open Challenges, Motivation and Recommendations. J. Med. Syst. 2018, 42, 80. [CrossRef]

5. Kim, J.; Chung, K.-Y. Ontology-based healthcare context information model to implement ubiquitous environment. Multimed. Tools Appl. 2011, 71, 873-888. [CrossRef]

6. Chen, T. Ubiquitous Multicriteria Clinic Recommendation System. J. Med. Syst. 2016, 40, 113. [CrossRef]

7. Zhao, P.; Yoo, I.; Lavoie, J.; Lavoie, B.J.; Simoes, E. Web-Based Medical Appointment Systems: A Systematic Review. J. Med. Internet Res. 2017, 19, e134. [CrossRef]

8. Chiu, M.-C.; Chen, T.-C.T. Assessing sustainable effectiveness of the adjustment mechanism of a ubiquitous clinic recommendation system. Health Care Manag. Sci. 2019, 23, 239-248. [CrossRef]

9. Tan, Y.Q.; Wu, Q.H.; Chiong, E. Preserving operational capability while building capacity during the COVID-19 pandemic: A tertiary urology centre's experience. Urology 2020, 142, 36-37. [CrossRef]

10. Flint, S.W.; Tahrani, A. COVID-19 and obesity-lack of clarity, guidance, and implications for care. Lancet Diabetes Endocrinol. 2020, 8, 474-475. [CrossRef]

11. Chen, T.-C.T. Ubiquitous clinic recommendation by predicting a patient's preferences. Electron. Commer. Res. Appl. 2017, 23, 14-23. [CrossRef]

12. Chen, T.-C.T.; Chiu, M.-C. A classifying ubiquitous clinic recommendation approach for forming patient groups and recommending suitable clinics. Comput. Ind. Eng. 2019, 133, 165-174. [CrossRef]

13. Ekstrand, M.D.; Riedl, J.T.; Konstan, J.A. Collaborative filtering recommender systems. Found. Trends Hum.-Comput. Interact. 2011, 4, 81-173. [CrossRef]

14. Dijs-Elsinga, J.; Otten, W.; Versluijs, M.M.; Smeets, H.J.; Kievit, J.; Vree, R.; Van Der Made, W.J.; De Mheen, P.J.M.-V. Choosing a Hospital for Surgery: The Importance of Information on Quality of Care. Med. Decis. Mak. 2010, 30, 544-555. [CrossRef]

15. Zwijnenberg, N.C.; Damman, O.C.; Spreeuwenberg, P.; Hendriks, M.; Rademakers, J.J. Different patient subgroup, different ranking? Which quality indicators do patients find important when choosing a hospital for hip- or knee arthroplasty? BMC Health Serv. Res. 2011, 11, 299. [CrossRef] [PubMed]

16. Tung, Y.-C.; Chang, G.-M. Patient satisfaction with and recommendation of a primary care provider: Associations of perceived quality and patient education. Int. J. Qual. Health Care 2009, 21, 206-213. [CrossRef]

17. Simanski, C.; Lefering, R.; Paffrath, T.; Riess, P.; Yücel, N.; Maegele, M.; Thüsing, C.; Neugebauer, E. Postoperative pain relief is an important factor for the patients' selection of a clinic. In Results of an Anonymous Survey; Schmerz: Berlin, Germany, 2006; pp. 327-333.

18. Bae, M.-R.; Chun, J.-H.; Lee, J.-H. Analysis of Selection Criteria of Consumers for Dental Clinic. J. Korea Contents Assoc. 2013, 13, 271-277. [CrossRef]

19. Lee, G.S. Study on selection factor in choosing dental clinic. J. Korean Acad. Dent. Technol. 2009, 31, 15-22.

20. Zulqarnain, R.M.; Abdal, S.; Ali, B.; Ali, L.; Dayan, F.; Ahamad, M.I.; Zafar, Z. Selection of medical clinic for disease diagnosis by using TOPSIS method. Int. J. Pharm. Sci. Rev. Res. 2020, 61, 22-27.

21. Chen, T. A FAHP-FTOPSIS approach for choosing mid-term occupational healthcare measures amid the COVID-19 pandemic. Health Policy Technol. 2021, 10, 100517. [CrossRef] 
22. Lee, W.-I.; Shih, B.-Y.; Chung, Y.-S. The exploration of consumers' behavior in choosing hospital by the application of neural network. Expert Syst. Appl. 2008, 34, 806-816. [CrossRef]

23. de Groot, I.B.; Otten, W.; Dijs-Elsinga, J.; Smeets, H.J.; Kievit, J.; Marang-van de Mheen, P.J.; CHOICE-2 Study Group. Choosing between hospitals: The influence of the experiences of other patients. Med. Decis. Mak. 2012, 32, 64-778. [CrossRef] [PubMed]

24. Ghosh, M. An empirical study on hospital selection in India. Int. J. Health Care Qual. Assur. 2015, 28, 27-39. [CrossRef]

25. Gündoğdu, F.K.; Kahraman, C.; Civan, H.N. A novel hesitant fuzzy EDAS method and its application to hospital selection. J. Intell. Fuzzy Syst. 2018, 35, 6353-6365. [CrossRef]

26. Alkadhi, O.H.; AlEissa, N.K.; AlMoharib, M.K.; Buquayyid, S.A. Influence of Social Media on the Patients for Choosing the Dental Clinic- A Cross-sectional Survey. J. Clin. Diagn. Res. 2020, 14, 32-34. [CrossRef]

27. Khoie, M.R.; Tabrizi, T.S.; Khorasani, E.S.; Rahimi, S.; Marhamati, N. A Hospital Recommendation System Based on Patient Satisfaction Survey. Appl. Sci. 2017, 7, 966. [CrossRef]

28. Paranjay, O.A.; Rajeshkumar, V. A Neural Network Aided Real-Time Hospital Recommendation System. Indones. J. Sci. Technol. 2020, 5, 217-235. [CrossRef]

29. Chao, H.; Cao, Y.; Zhang, J.; Xia, F.; Zhou, Y.; Shan, H. Population Density-Based Hospital Recommendation with Mobile LBS Big Data. In Proceedings of the 2018 IEEE International Conference on Big Data and Smart Computing (BigComp), Shanghai, China, 15-17 January 2018; pp. 37-44.

30. Tawarmalani, M.; Sahinidis, N.V. Convexification and Global Optimization in Continuous and Mixed-Integer Nonlinear Programming: Theory, Algorithms, Software, and Applications; Kluwer Academic Publishers: Dordrecht, The Netherlands, 2002.

31. Chen, T.-C.T.; Chiu, M.-C. Mining the preferences of patients for ubiquitous clinic recommendation. Health Care Manag. Sci. 2018, 23, 173-184. [CrossRef]

32. Kulkarni, A.; Vishwanath, A.; Shah, C. Implementing a Real-Time, AI-Based, Face Mask Detector Application for COVID-19. Available online: https://developer.nvidia.com/blog/implementing-a-real-time-ai-based-face-mask-detector-application-forcovid-19/ (accessed on 8 September 2021).

33. Collado-Borrell, R.; Escudero-Vilaplana, V.; Villanueva-Bueno, C.; Herranz-Alonso, A.; Sanjurjo-Saez, M. Features and Functionalities of Smartphone Apps Related to COVID-19: Systematic Search in App Stores and Content Analysis. J. Med. Internet Res. 2020, 22, e20334. [CrossRef]

34. Wu, H.-C.; Chen, T. CART-BPN approach for estimating cycle time in wafer fabrication. J. Ambient. Intell. Humaniz. Comput. 2014, 6, 57-67. [CrossRef]

35. Tealab, A.; Hefny, H.; Badr, A. Forecasting of nonlinear time series using ANN. Futur. Comput. Inform. J. 2017, 2, 39-47. [CrossRef]

36. Chen, T.; Wang, Y.-C.; Tsai, H.-R. Lot cycle time prediction in a ramping-up semiconductor manufacturing factory with a SOM-FBPN-ensemble approach with multiple buckets and partial normalization. Int. J. Adv. Manuf. Technol. 2009, 42, 1206-1216. [CrossRef]

37. Nocedal, J.; Wright, S.J. Numerical Optimization; Springer: New York, NY, USA, 1999.

38. Wang, Y.-C.; Tsai, H.-R.; Chen, T. A Selectively Fuzzified Back Propagation Network Approach for Precisely Estimating the Cycle Time Range in Wafer Fabrication. Mathematics 2021, 9, 1430. [CrossRef]

39. Chen, T.; Wang, Y.-C. Estimating simulation workload in cloud manufacturing using a classifying artificial neural network ensemble approach. Robot. Comput. Manuf. 2016, 38, 42-51. [CrossRef]

40. Wang, Y.-C.; Chen, T.; Hsu, T.C. A fuzzy deep predictive analytics approach for enhancing cycle time range estimation precision in wafer fabrication. Decis. Anal. J. 2021, 1, 100010. [CrossRef]

41. Bianco, S.; Cadene, R.; Celona, L.; Napoletano, P. Benchmark Analysis of Representative Deep Neural Network Architectures. IEEE Access 2018, 6, 64270-64277. [CrossRef]

42. Chen, T.-C.T.; Tsai, H.-R. Multilayer fuzzy neural network for modeling a multisource uncertain unit-cost learning process in wafer fabrication. Rapid Prototyp. J. 2018, 24, 521-531. [CrossRef]

43. Tang, J.; Zhang, X.; Yin, W.; Zou, Y.; Wang, Y. Missing data imputation for traffic flow based on combination of fuzzy neural network and rough set theory. J. Intell. Transp. Syst. 2020, 25, 439-454. [CrossRef]

44. Al-Refaie, A.; Chen, T.; Al-Athamneh, R.; Wu, H.-C. Fuzzy neural network approach to optimizing process performance by using multiple responses. J. Ambient. Intell. Humaniz. Comput. 2016, 7, 801-816. [CrossRef]

45. Khatter, H.; Ahlawat, A.K. An intelligent personalized web blog searching technique using fuzzy-based feedback recurrent neural network. Soft Comput. 2020, 24, 9321-9333. [CrossRef] 